\title{
Outcome Predictors in Acute Basilar Artery Occlusion
}

\author{
Tomáš Dorňák, Roman Herzig, David Školoudík, Daniel Šaňák, Martin Kuliha, \\ Martin Roubec, Martin Köcher, Václav Procházka, Michal Král, Tomáš Veverka, \\ Petr Hluštík, Jana Zapletalová, Miroslav Heřman
}

\begin{abstract}
Objective: To identify predictors of good outcome in acute basilar artery occlusion (BAO). Background: Acute ischemic stroke (AIS) caused by BAO is often associated with a severe and persistent neurological deficit and a high mortality rate. Methods: The set consisted of 70 consecutive AIS patients (51 males; mean age $64.5 \pm 14.5$ years) with BAO. The role of the following factors was assessed: baseline characteristics, stroke risk factors, pre-event antithrombotic treatment, neurological deficit at time of treatment, estimated time to therapy procedure initiation, treatment method, recanalization rate, change in neurological deficit, post-treatment imaging findings. 30- and 90-day outcome was assessed using the modified Rankin scale with a good outcome defined as a score of 0 3. Results: The following statistically significant differences were found between patients with good versus poor outcomes: mean age (54.2 vs. 68.9 years; $p=0.0001)$, presence of arterial hypertension $(52.4 \%$ vs. $83.7 \% ; p=0.015)$, diabetes mellitus $(9.5 \%$ vs. $55.1 \%$; $p=0.0004)$ and severe stroke $(14.3 \%$ vs. $65.3 \% ; p=0.0002)$, neurological deficit at time of treatment $(14.0$ vs. 24.0 median of National Institutes of Health Stroke Scale [NIHSS] points; $p=0.001)$, successful recanalization $(90.0 \%$ vs. 54.2\%; $p=0.005)$, change in neurological deficit (12.0 vs. 1.0 median difference of NIHSS points; $p=0.005)$. Stepwise binary logistic regression analysis identified age $(\mathrm{OR}=0.932,95 \% \mathrm{CI}=0.882-0.984 ; p=0.012)$, presence of diabetes mellitus $(\mathrm{OR}=0.105,95 \% \mathrm{CI}=0.018-0.618 ; p=0.013)$ and severe stroke $(\mathrm{OR}=0.071,95 \% \mathrm{CI}=0.013-0.383 ; p=0.002)$ as significant independent negative predictors of good outcome. Conclusions: In the present study, higher age, presence of diabetes mellitus and severe stroke were identified as significant independent negative predictors of good outcome.
\end{abstract}

RÉSUMÉ: Facteurs de prédiction du résultat dans l'occlusion aiguë de l'artère basilaire. Objectif : Le but de l'étude était d'identifier les facteurs de prédiction d'une issue favorable dans l'occlusion aiguë de l'artère basilaire $(\mathrm{OAB})$. Contexte : L'accident vasculaire cérébral ischémique aigu (AVCIA) causé par une $\mathrm{OAB}$ est souvent associé à un déficit neurologique sévère et persistant et à un taux de mortalité élevé. Méthodes : L'étude a porté sur 70 patients consécutifs atteints d'une OAB, dont 51 étaient des hommes et dont l'âge moyen était de 64,5 \pm 14,5 ans. Nous avons étudié le rôle des facteurs suivants : les caractéristiques des patients à l'évaluation initiale, les facteurs de risque d'un AVC, le traitement antithrombotique avant l'OAB, le déficit neurologique au moment du traitement, le temps écoulé entre le début des symptômes et le début du traitement, le mode de traitement, le taux de recanalisation, les changements constatés concernant le déficit neurologique, les constatations à l'imagerie après le traitement. Nous avons évalué les résultats à deux reprises, soit 30 jours et 90 jours après l'événement, au moyen de l'échelle modifiée de Rankin, une issue favorable étant définie comme un score de 0 à 3 . Résultats : Les différences suivantes étaient significatives au point de vue statistique entre les patients dont l'issue était bonne par rapport à ceux dont l'issue était médiocre : l'âge moyen des patients $(54,2$ vs. 68,9 ans ; p = 0,0001), la présence d'hypertension artérielle $(52,4 \%$ vs. $83,7 \% ; p=0,015)$, de diabète $(9,5 \%$ vs. $55,1 \% ; p=0,0004)$ et d'un AVC sévère $(14,3 \%$ vs. $65,3 \% ; p=0,0002)$, le déficit neurologique au moment du traitement (score médian au NIHSS 14,0 versus 24,$0 ; p=0,001)$, une recanalisation réussie $(90,0 \%$ vs. $54,2 \% ; p=0,005)$, le changement du déficit neurologique (médiane de la différence du score NIHSS 12,0 vs. $1 ; \mathrm{p}=0,005$ ). L'analyse de régression logistique binaire pas à pas a montré que l'âge $(\mathrm{RC}=0,932$; IC à $95 \%: 0,882$ à 0,$984 ; \mathrm{p}=0,012)$, la présence de diabète $(\mathrm{RC}=0,105 ; \mathrm{IC}$ à $95 \%: 0,018$ à $0.618 ; \mathrm{p}=0,013)$ et la sévérité de l'AVC ( $\mathrm{RC}=0,071$; IC à 95\% : 0,013 à 0,$383 ; \mathrm{p}=0,002)$ étaient des facteurs de prédiction négatifs indépendants d'une issue favorable. Conclusions : Dans cette étude, un âge plus avancé, la présence de diabète et la sévérité de l'AVC ont été identifiés comme étant des facteurs négatifs indépendants significatifs d'une issue favorable.

Can J Neurol Sci. 2014; 41: 368-374

Basilar artery occlusion (BAO) is an infrequent form of acute ischemic stroke (AIS). This particular type of stroke occurs in approximately $1 \%$ of all-cause cases and is reported in approximately $8 \%$ of patients with symptomatic vertebrobasilar territory ischemia. ${ }^{1,2}$ Basilar artery occlusion symptoms are heterogeneous and may vary from mild neurological deficits consisting of visual, oculomotor, and behavioral abnormalities to a locked-in state or coma. ${ }^{3,4}$ However, severe and persistent
From the Departments of Neurology (TD, RH, DŠk, DŠa, MKu, MKr, TV, PH) and Radiology (MKö, MH), Faculty of Medicine and Dentistry, Palacký University and University Hospital, Olomouc; Department of Medical Biophysics (JZ), Faculty of Medicine and Dentistry, Institute of Molecular and Translational Medicine, Palacký University, Olomouc; Departments of Neurology (DŠk, MKu, MR) and Radiology (VP), University Hospital and Ostrava University, Ostrava-Poruba, Czech Republic.. Received July 16, 2013. Final Revisions Submitted November 27, 2013. Correspondence to: Roman Herzig, Department of Neurology, Faculty of Medicine and Dentistry, Palacký University and University Hospital, I. P. Pavlova 6, CZ-775 20 Olomouc, Czech Republic. Email: herzig.roman@seznam.cz. 
neurologic deficits and a high mortality rate can occur in spite of recent advances in the treatment of AIS ${ }^{5}$ Moreover, patients with $\mathrm{BAO}$ are on average nearly a decade younger than patients with all-cause AIS. ${ }^{1,2}$ Acute ischemic stroke due to BAO occurs also in children, with an estimated incidence of 0.037 per 100,000 children per year. ${ }^{6-8}$

Several studies, including a meta-analysis and registry-based investigation, have evaluated the treatment responses following different approaches in the management of AIS. ${ }^{9-12}$ Also, in acute BAO, most studies have focused on an assessment of the association between clinical outcome or recanalization rate and type of therapy. For example, the results of the Basilar Artery International Cooperation Study (BASICS) registry suggest that the effectiveness of treatment modalities for patients with mildto-moderate deficits may differ. Nevertheless, no statistically significant superiority existed for any treatment strategy, i.e., antithrombotic treatment (AT) only, intravenous thrombolysis (IVT), and intra-arterial treatment (IAT).$^{11}$

The role of other factors influencing the outcome of acute BAO patients has not been widely studied. Assessment of the role of such risk factors using data from daily clinical practice should extend the knowledge in this field and their identification may be helpful when considering the use of various treatment strategies (including invasive and less available intra-arterial and bridging therapy) and also when designing controlled randomized trials.

The aim of the present study was to identify predictors of good clinical outcome in AIS patients with BAO.

\section{METHODS}

\section{Patients and inclusion criteria}

A bi-centric retrospective study was performed, using data from hospital registries of all ischemic stroke patients. Seventy consecutive AIS patients with BAO admitted to the Comprehensive Stroke Center of the University Hospital Olomouc and to the Comprehensive Stroke Center of the University Hospital Ostrava (Czech Republic) between January 2006 and May 2013 were included in the study. All patients met the following inclusion criteria: neurologic deficit attributable to the posterior circulation; and BAO confirmed by time-of-flight magnetic resonance angiography (TOF MRA) or computed tomography angiography (CTA). There were no exclusion criteria.

\section{Data abstraction}

Data was gathered from medical records and patient histories, including baseline characteristics, risk factors, pre-event AT, neurologic deficits at the time of treatment, type and estimated time to initiation of therapy, recanalization rate, change in neurologic deficits (difference between admission and discharge), post-treatment imaging findings, and clinical outcomes on days 30 and 90 .

The existence of the following stroke risk factors (either previously known or newly diagnosed) was noted: arterial hypertension; diabetes mellitus; hyperlipidemia; atrial fibrillation; coronary artery disease; smoking history; recent neck trauma; and dissection of the vertebral artery.
At the time of treatment, neurologic deficits were assessed with the National Institutes of Health stroke scale (NIHSS). Separately and in agreement with the BASICS trial ${ }^{11}$, stroke severity was defined as severe (coma, locked-in state, and tetraplegia) or mild-to-moderate (other than severe).

The estimated time of BAO was considered as the time of symptom onset attributable to dysfunction in the posterior circulation as reported by the patient or witnesses. In cases in which the exact time of BAO remained unclear, the time when the patient was last seen by a witness before symptom onset was used. Minor stroke or transient ischemic attack (TIA) was recorded separately under the prodromal phase.

The type of therapy was at the discretion of the treating physician. The following treatments were used: 1) AT, including antiplatelet drugs or systemic anticoagulation, both of which were used strictly according to the valid European Stroke Organisation guidelines ${ }^{13,14}$; 2) IVT alone with recombinant tissue plasminogen activator (rt-PA [Actilyse $\AA$; Boehringer Ingelheim, Ingelheim am Rhein, Germany]); 3) IVT with subsequent IAT; or 4) IAT alone. Intra-arterial treatment was comprised of intra-arterial thrombolysis with rt-PA, percutaneous transluminal angioplasty (PTA), mechanical thrombectomy (Solitaire ${ }^{\mathrm{TM}} \mathrm{AB}$; ev3 Inc., Irvine, CA, USA), stenting (Wingspan ${ }^{\mathrm{TM}}$ Stent System with Gateway ${ }^{\mathrm{TM}}$ PTA Balloon Catheter; Boston Scientific Corporation, Natick, MA, USA), endovascular sono-lysis (Micro Lys US Sonography Catheter; EKOS Corporation, Bothell, WA, USA), or a combination of these methods. In each of the four groups, the time-to-therapy was recorded. The time-to-intervention was defined as the time from symptom onset to IVT start, both in the IVT and IVT+IAT groups (IVT was the first therapy administered to the patients in the latter group as well), and as a time from symptom onset-to-groin puncture in the IAT group. In the AT group, the time-to-intervention was defined as the time from symptom onset to the first intake of antithrombotic medication. The time of symptom onset was known for all patients.

Clinical outcome both on days 30 and 90 was evaluated using the modified Rankin scale (mRS), with a good clinical outcome defined as a score of $0-3$. This definition was used according to previous studies that assessed clinical outcome in BAO, ${ }^{1,11,15}$ and taking into account the naturally severe course of this type of stroke. Evaluation of mRS was performed during follow-up clinical visits by certified neurologists and entered in the patient medical record.

Post-treatment imaging was performed by computed tomography (CT) or magnetic resonance imaging (MRI) and was carried out within 24 hours (h) after treatment initiation. The presence of hemorrhagic and ischemic changes was assessed by a certified radiologist and systematically recorded. Symptomatic intracranial hemorrhage was defined as any CT- or MRIdocumented hemorrhage that was temporally related to deterioration in the patient's clinical condition according to the judgment of the clinician. ${ }^{16}$ Recanalization in post-treatment angiograms was dichotomized using the Thrombolysis in Cerebral Infarction (TICI) score $^{17}$ as complete-to-partial (TICI 2-3), and minimal-to-nil (TICI 0-1). The TICI score was generated at the end of the angiographic procedure in IVT+IAT and IAT patients by a certified radiologist. In the IVT group, 


\section{Table 1: Patient characteristics}

\begin{tabular}{lll}
\hline Observed factor & All $(\mathrm{n}=70)$ & $\%$ \\
\hline Age (mean \pm SD) & $64.5 \pm 14.5$ & \\
Males & 51 & 72.9 \\
Presence of & 52 & 74.3 \\
$\quad$ Arterial hypertension & 27 & 38.6 \\
$\quad$ Coronary artery disease & 15 & 21.4 \\
$\quad$ Atrial fibrillation & 29 & 41.4 \\
$\quad$ Diabetes mellitus & 31 & 44.3 \\
$\quad$ Hyperlipidemia & 16 & 22.9 \\
$\quad$ Smoking history & 23 & 32.9 \\
Pre-event AT & & \\
Treatment method & 10 & 14.3 \\
$\quad$ AT & 20 & 28.6 \\
$\quad$ IVT & 18 & 25.7 \\
IVT+IAT & 22 & 31.4 \\
IAT & & \\
Deficit at time of treatment & 35 & 50.0 \\
$\quad$ Mild to moderate & 35 & 50.0 \\
Severe & $20.5 \pm 9.0$ & \\
$\quad$ NIHSS score (mean \pm SD) & &
\end{tabular}

AT: antithrombotic therapy, IAT: primary intra-arterial therapy, IVT: primary intravenous thrombolysis, IVT+IAT: intravenous thrombolysis with a subsequent intra-arterial therapy, NIHSS: National Institutes of Health Stroke Scale, SD: standard deviation.

CTA, MRA, or transcranial Doppler (TCD) were carried out (up to $24 \mathrm{~h}$ ) after treatment, and recanalization rate was assessed by a certified radiologist (CTA and MRA) or certified neurosonologist (TCD). In CTA, MRA, and TCD, recanalization was assessed as complete-to-partial or minimal-to-nil. The results were also reviewed by two neurologists and radiologists and no disagreements were noted. The assessing physicians were not blinded.

\section{Ethics}

The entire study was conducted in accordance with the Helsinki Declaration of 1975 (as revised in 2004 and 2008), and was approved by the Local Ethics Committees of participating hospitals (University Hospital Olomouc and University Hospital Ostrava). All subjects gave informed consent.

\section{Statistical analysis}

The normality of the distributions was confirmed using the Shapiro-Wilk test. Fisher's exact test was used to assess relationship between clinical outcome and observed categorical parameters. The Mann-Whitney U-test or unpaired t-test was used, depending on data normality, to evaluate the impact of quantitative parameters. Forward stepwise binary logistic regression, including all variables with a $p<0.05$ after univariate analysis (age, presence of arterial hypertension and diabetes mellitus, presence of severe neurologic deficits, NIHSS at the time of treatment, and recanalization rate), was used to determine the significant predictors of good clinical outcome. Tests were carried out at a significance level of $p<0.05$. Statistical analysis was performed using SPSS 15 (SPSS Inc., Chicago, IL, USA). Missing data was not included into statistical analysis.

\section{RESULTS}

Seventy patients (51 males; mean age, $64.5 \pm 14.5$ years) from two participating centers were included in the study. Basilar artery occlusion was confirmed using CTA alone in 61 patients, MRA with subsequent CTA or digital subtraction angiography in seven patients, and MRA alone in two patients. None of the patients had recent neck trauma or dissection of the vertebral artery.

Ten (14.3\%) patients were treated with AT, 22 (31.4\%) were treated with IAT, $20(28.6 \%)$ were treated with IVT, and 18 (25.7\%) were treated with combined IVT and IAT. In the IAT group, six received intra-arterial thrombolysis only, four received mechanical thrombectomy only, three received PTA only, one received stenting only, and eight were treated with a combination of these methods. In the IVT+IAT group, four patients were treated with subsequent local thrombolysis, five were treated with mechanical thrombectomy, one was treated with stenting, one was treated with sonothrombolysis, one was treated with PTA, and a combination of these methods was used in six patients. Table 1 shows the patient characteristics.

Partial or complete recanalization was observed in $6(31.6 \%)$ IVT patients, $16(88.8 \%)$ IVT+IAT patients, 21 (90.9\%) IAT patients, and $2(22.2 \%)$ AT patients. Symptomatic hemorrhage was found in $12.9 \%$ of patients.

Thirty day and 90-day clinical outcome is demonstrated in the Figure.

Good clinical outcomes were noted in $21(30.0 \%)$ patients (two [20.0\%] AT patients, five [25.0\%] IVT patients, nine [50.0\%] IVT+IAT patients, and five [22.7\%] IAT patients; $p=0.216$ ) and they did not differ between day 30 and day 90 . The overall mortality rate was $34.3 \%$ (24 patients) on day 30 and $44.3 \%$ (31 patients) on day 90. Table 2 demonstrates the differences between patients with good versus poor outcomes. The following statistically significant differences were found between patients with good versus poor outcomes: mean age (54.2 vs. 68.9 years; $p=0.0001$ ); presence of arterial hypertension $(52.4 \%$ vs. $83.7 \%$; $p=0.015)$ and diabetes mellitus $(9.5 \%$ vs. $55.1 \% ; p=0.0004)$; presence of severe stroke $(14.3 \%$ vs. $65.3 \% ; p=0.0002)$; neurologic deficit at the time of treatment (14.0 vs. 24.0 median of NIHSS points; $p=0.001$ ); successful recanalization ( $90.0 \%$ vs. $54.2 \% ; p=0.005)$; and change in neurologic deficits (12.0 vs. 1.0 median difference of NIHSS points; $p=0.007$ ).

Based on univariate logistic regression analysis, age ( $\mathrm{OR}=0.920,95 \% \mathrm{CI}=0.878-0.964 ; p=0.0005)$, presence of arterial hypertension $(\mathrm{OR}=0.215,95 \% \quad \mathrm{CI}=0.068-0.673$; $p=0.008)$ and diabetes mellitus (OR=0.086, 95\% CI $=0.018$ $0.409 ; p=0.002)$, presence of severe stroke $(\mathrm{OR}=0.089,95 \% \mathrm{CI}=$ $0.023-0.344 ; p=0.0005)$, NIHSS value at the time of treatment $(\mathrm{OR}=0.903,95 \% \mathrm{CI}=0.846-0.963 ; p=0.002)$, and recanalization rate $(\mathrm{OR}=7.615,95 \% \mathrm{CI}=1.589-36.51 ; p=0.011)$ were identified as significant predictors of clinical outcome, as presented in Table 3.

Stepwise binary logistic regression analysis identified higher age $(\mathrm{OR}=0.932,95 \% \mathrm{CI}=0.882-0.984 ; p=0.012)$, presence of diabetes mellitus $(\mathrm{OR}=0.105,95 \% \mathrm{CI}=0.018-0.618 ; p=0.002)$, 


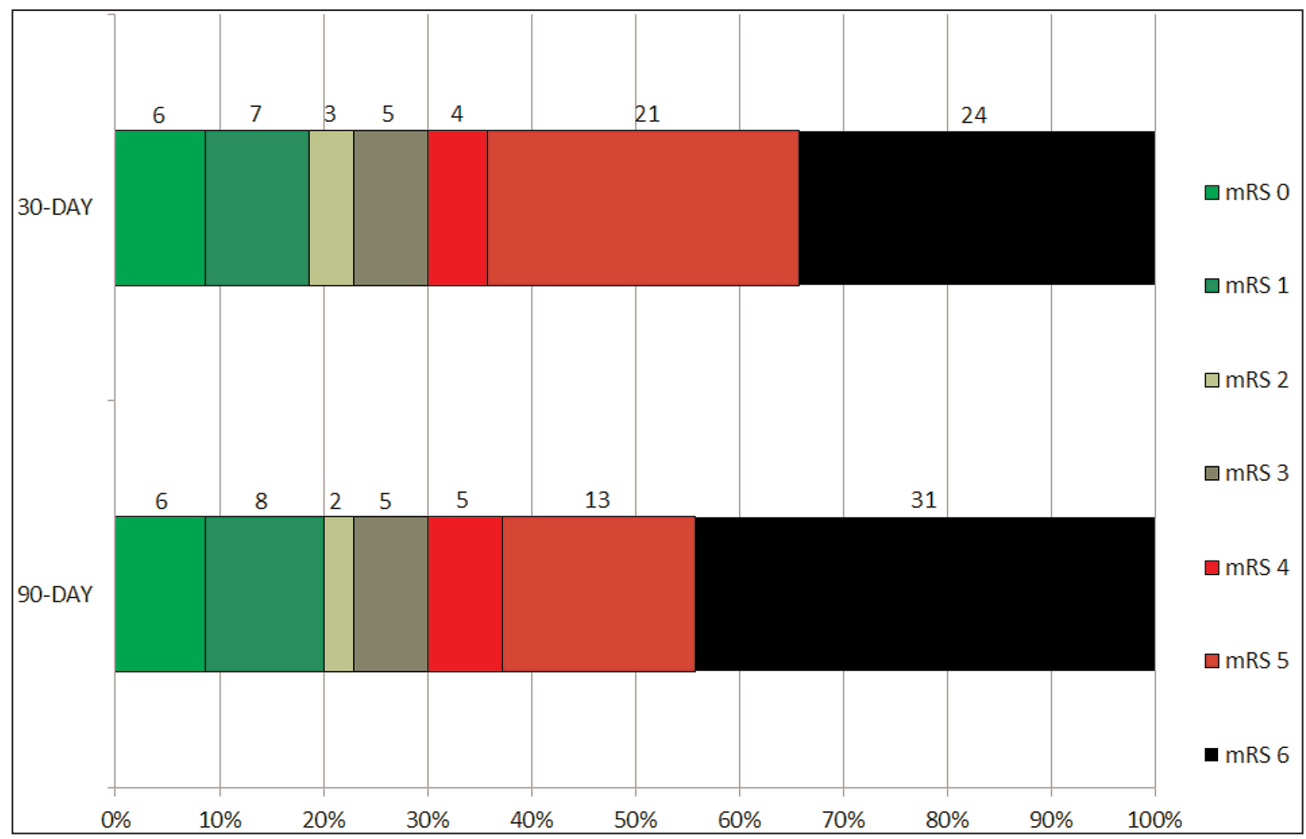

Figure: 30-day and 90-day clinical outcome.

and presence of severe stroke $(\mathrm{OR}=0.071,95 \% \mathrm{CI}=0.013-0.383$; $p=0.002$ ) as significant independent negative predictors of good outcome.

\section{Discussion}

Most previously published studies performed in patients with acute BAO assessed the association between clinical outcome or recanalization rate and type of therapy. Nevertheless, the role of other factors has not been widely studied.

Age was identified as a significant independent predictor of good 30- and 90-day clinical outcomes in the present study and of long-term ( $\geq 12$ months) clinical outcome in the study performed by Jung et $a l^{9}$ No significant association between gender and clinical outcome was observed, which is in agreement with the results of previous studies. ${ }^{18,19}$

The presence of arterial hypertension was identified as a predictor of clinical outcome based only on univariate analysis, but not multivariate logistic regression analysis in the present study and the study conducted by Jung et $a l^{9}$, who reported the influence on not only three month survival, but also long-term survival. In contrast, the presence of diabetes mellitus was identified as a significant independent negative predictor of good outcome in both studies.

In agreement with other reports ${ }^{10,20-22}$, a strong coherence between pre-treatment stroke severity and resulting clinical outcome was demonstrated in the present study. Jung et al ${ }^{9}$ identified low baseline NIHSS score as a significant predictor of good or moderate clinical outcome and survival at three months. In the current study, the presence of severe stroke was identified as a significant independent negative predictor of good 30- and 90-day outcomes.
Controversial results regarding the possible association between time-to-treatment with clinical outcome in patients with BAO have been published previously. Specifically, no significant association was reported in the studies conducted by Nagel et $a l^{15}$, Strbian et $a l^{23}$, and the present study (time-totreatment, 3.9 versus 4.3 hours in patients with good vs. poor outcome; $p=0.676$ ). In contrast, when sub-analysing data from the BASICS trial, it was found that early recanalization therapy in patients with $\mathrm{BAO}$ is associated with a more favorable outcome with a significantly increased chance of a poor outcome when recanalization therapy was started $>6 \mathrm{~h}$ after the estimated time of $\mathrm{BAO} .^{24}$ Thus, the role of time-to-treatment remains unclear in BAO in contrast to the anterior circulation.

Recanalization of BAO is the main goal of every therapeutic strategy. Schonewille et $a l^{11}$ demonstrated that successful recanalization represents a protective factor against poor outcomes in patients treated with IVT $(R R=0.67)$ and IAT $(\mathrm{RR}=0.75)$. In the present study, partial or complete recanalization was achieved in $62.9 \%$ patients, and it was found significantly more frequently in patients with good $(94.1 \%)$ versus poor $(52.3 \%)$ outcomes. According to previously published results, the efficacy of the particular treatment methods differs. For example, in a recent study Sairanen et al ${ }^{10}$ achieved recanalization in $65 \%$ of patients undergoing IVT, reaching the efficacy of IAT which appears to be the most effective method, with a $62.5 \%-83.7 \%$ recanalization rate. ${ }^{9,11,15}$ Apart from these studies comprising $>100$ patients, several smaller studies exist which have evaluated the recanalization rate for different types of IAT, reaching a recanalization rate up to $94 \%$ using local thrombolysis and additional balloon angioplasty, and up to $77 \%$ using local thrombolysis and stent 
Table 2: Differences between patients with good versus poor outcome (both on day 30 and day 90)

\begin{tabular}{|c|c|c|c|}
\hline \multirow[t]{2}{*}{ Observed factor } & \multicolumn{2}{|c|}{ Clinical outcome } & \multirow[t]{2}{*}{$p$-value } \\
\hline & $\begin{array}{c}\text { Good } \\
(\mathrm{mRS} \mathrm{0-3)} \\
\mathrm{n}=21\end{array}$ & $\begin{array}{c}\text { Poor } \\
\text { (mRS 4-6) } \\
\mathrm{n}=49\end{array}$ & \\
\hline Age & $54.2(13.2)$ & $68.9(12.8)$ & $0.0001 *$ \\
\hline Male gender & $16(76.2 \%)$ & $35(71.4 \%)$ & 0.776 \\
\hline \multicolumn{4}{|l|}{ Presence of } \\
\hline Arterial hypertension & $11(52.4 \%)$ & $41(83.7 \%)$ & $0.015 *$ \\
\hline Coronary artery disease & $5(23.8 \%)$ & $22(44.9 \%)$ & 0.115 \\
\hline Atrial fibrillation & $4(19.0 \%)$ & $11(22.9 \%)$ & 1.000 \\
\hline Diabetes mellitus & $2(9.5 \%)$ & $27(55.1 \%)$ & $0.0004 *$ \\
\hline Hyperlipidemia & $8(38.1 \%)$ & $23(48.9 \%)$ & 0.442 \\
\hline Smoking history & $6(33.3 \%)$ & $10(26.3 \%)$ & 0.752 \\
\hline Pre-event AT & $5(23.8 \%)$ & $18(37.5 \%)$ & 0.406 \\
\hline Pre-event mRS [mean (min-max)] & $0(0-1)$ & $0(0-4)$ & 0.428 \\
\hline Severe deficit at time of treatment & $3(14.3 \%)$ & $32(65.3 \%)$ & $0.0002 *$ \\
\hline NIHSS at time of treatment & $14.0(7.5-20.0)$ & $24.0(16.0-30.0)$ & $0.001^{*}$ \\
\hline Time to treatment (hours) & $4.2(2.4-7.3)$ & $4.6(3.0-7.8)$ & 0.160 \\
\hline Treatment method & & & 0.235 \\
\hline AT & $2(9.5 \%)$ & $8(16.3 \%)$ & \\
\hline IVT & $5(23.8 \%)$ & $15(30.6 \%)$ & \\
\hline IVT+IAT & $9(42.9 \%)$ & $9(18.4 \%)$ & \\
\hline IAT & $5(23.8 \%)$ & $17(34.7 \%)$ & \\
\hline Successful recanalization & $18(90.0 \%)$ & $26(54.2 \%)$ & $0.005^{*}$ \\
\hline Change in NIHSS & $12.0(5.5-15.5)$ & $1.0(-0.3-10.8)$ & $0.007^{*}$ \\
\hline \multicolumn{4}{|l|}{ Post treatment imaging changes } \\
\hline Hemorrhagic & $1(4.8 \%)$ & $7(18.4 \%)$ & 0.238 \\
\hline Ischemic & $12(60.0 \%)$ & $31(81.6 \%)$ & 0.114 \\
\hline
\end{tabular}

Data is mean $(\mathrm{SD})$, number $(\%)$, or median $\left(25^{\text {th }}-75^{\text {th }}\right.$ percentile $)$, unless otherwise stated. ${ }^{*} \mathrm{p}<0.05$; AT: antithrombotic therapy, IAT: primary intra-arterial therapy, IVT: primary intravenous thrombolysis, IVT+IAT: intravenous thrombolysis with a subsequent intra-arterial therapy, max: maximal, min: minimal, mRS: modified Rankin scale, NIHSS:

National Institutes of Health Stroke Scale.

Table 3: Results of binary logistic regression analysis (unadjusted OR) - dependent variable (30-day and 90-day mRS 0-3)

\begin{tabular}{lccc}
\hline Observed factor & OR & $95 \%$ CI & $p$-value \\
\hline Age & 0.920 & $0.878-0.964$ & $0.0005^{*}$ \\
Male gender & 0.781 & $0.240-2.543$ & 0.682 \\
Presence of & & & $0.008^{*}$ \\
$\quad$ Arterial hypertension & 0.215 & $0.068-0.673$ & 0.103 \\
$\quad$ Coronary artery disease & 0.384 & $0.121-1.213$ & 0.720 \\
$\quad$ Atrial fibrillation & 0.791 & $0.220-2.848$ & $0.002^{*}$ \\
$\quad$ Diabetes mellitus & 0.086 & $0.018-0.409$ & 0.408 \\
$\quad$ Hyperlipidemia & 0.642 & $0.225-1.835$ & 0.588 \\
$\quad$ Smoking history & 1.400 & $0.414-4.729$ & 0.271 \\
Pre-event AT & 1.521 & $0.163-1.664$ & 0.324 \\
Pre-event mRS & 0.492 & $0.120-2.018$ & $0.0005^{*}$ \\
Severe neurological deficit at time of treatment & 0.089 & $0.023-0.344$ & $0.002^{*}$ \\
NIHSS at time of treatment & 0.903 & $0.846-0.963$ & 0.197 \\
Time to treatment (hours) & 0.198 & $0.017-2.313$ & 0.216 \\
Treatment method & & & 0.761 \\
$\quad$ IAT vs. AT & 1.333 & $0.209-8.49$ & 0.132 \\
$\quad$ IVT vs. AT & 4.000 & $0.659-24.30$ & 0.863 \\
$\quad$ IVT+IAT vs. AT & 1.176 & $0.186-7.43$ & $0.011^{*}$ \\
Recanalization rate & 7.615 & $1.589-36.51$ & 0.173 \\
Post treatment imaging changes & & & 0.080 \\
$\quad$ Hemorrhagic & 0.221 & $0.025-1.938$ & $0.101-1.140$ \\
$\quad$ Ischemic & 0.339 & &
\end{tabular}

${ }^{*} \mathrm{p}<0.05$; AT: antithrombotic therapy, CI: confidence interval, IAT: primary intra-arterial therapy, IVT: primary intravenous thrombolysis, IVT+IAT: intravenous thrombolysis with a subsequent intra-arterial therapy, mRS: modified Rankin scale, NIHSS: National Institutes of Health Stroke Scale, OR: odds ratio. 
placement. ${ }^{25,26}$ In the present study, partial or complete recanalization was observed in $26.6 \%$ of patients treated with IVT, $88.2 \%$ of patients treated with IVT+IAT, and $90.0 \%$ of patients treated with IAT.

An apparent, although statistically insignificant trend for better clinical outcome was observed in patients treated with combination of IVT and IAT in the present study. It is rather curious that despite the very high recanalization rate in the IAT group and the low recanalization rate in the IVT group, both groups have almost the same resulting good clinical outcome ( $20.0 \%$ in the IAT group vs. $26.7 \%$ in the IVT group), which may be explained by different methods used for evaluation of the recanalization rate in the IVT and IAT groups. Lindsberg et al ${ }^{12}$ and the BASICS trial ${ }^{11}$ also indicated that efficacy of IVT and IAT for treatment of BAO may be similar. Nevertheless, no comparisons have been published to date regarding IVT versus IVT+IAT for BAO. A single study comprised of 52 patients $^{27}$ compared patients with BAO who were treated with IAT versus IVT+IAT. The results reported in that study are comparable to the results in the current study $(50.0 \%$ vs. $47.1 \%$ of IVT+IAT patients and $23.0 \%$ vs. $20.0 \%$ of IAT patients reaching good $90-$ day clinical outcomes [defined as a mRS score of 0-3]).

The number of patients who achieved a good clinical outcome did not differ between days 30 and 90 in the present set, but one may assume that some patients might improve during a longer follow-up period, as reported in two large studies., ${ }^{9,28}$ None of the patients in the present set improved from "poor clinical outcome" to "good clinical outcome" and vice versa. In the study conducted by Jung et $a l^{9}, 40 \%$ of patients with BAO presented with clinical improvement of at least 1 point on the mRS when comparing three month and long-term ( $\geq 12$ months) outcomes. Individuals with mRS scores of 2-4 often improve, while patients with the most severe disability at three months (mRS score of 5) do not. ${ }^{28}$ In the present study, the overall mortality (34.3\% on day 30 and $44.3 \%$ on day 90) was influenced by the withdrawal of care, which was used only in patients with a mRS score of 5 .

Post-treatment imaging was performed in $83.9 \%$ of the patients; the remainder of the patients either presented with a severe neurologic state (one patient) or died (nine patients) within $24 \mathrm{~h}$ from treatment onset. As a consequence of using $\mathrm{mRS}$, the post-treatment imaging result was not taken into account when assessing clinical outcome. Nevertheless, it was assessed along with ischemic changes separately as a factor possibly influencing clinical outcome. Symptomatic hemorrhage was found in $12.9 \%$ of patients. Hemorrhagic complications occurred more frequently after IAT $(20.0 \%)$ than after IVT $(6.7 \%)$ or IVT+IAT $(11.8 \%)$. In the BASICS registry, the rate of symptomatic intracranial hemorrhage was $14 \%$ after IAT and $6 \%$ after IVT. These results suggest that use of intravenous rt-PA is safer than bridging therapy with abciximab which has higher rates of symptomatic hemorrhage $(14 \%-15 \%){ }^{15,29}$ Published results reinforce the use of IVT+IAT as a therapeutic approach in patients with documented arterial occlusion and the need to shorten the time-to-treatment and, in this context, the additional IAT approach probably should be started as soon as possible and not considered only as a rescue strategy. ${ }^{30}$

Several limitations of the present study should be mentioned. The retrospective observational study represents one of them.
Second, the choice of the treatment method was dependent on physician decisions lacking any treatment protocol. Third, the limited number of included patients did not allow us to reach statistically significant differences between the particular treatment groups, although an apparent trend for better clinical outcomes was observed in the combined IVT+IAT group. Also, in two patients from our registry, BAO was diagnosed using MRA only. It should be mentioned that MRA is less sensitive than other modalities and in situations of slow and residual flow may show discontinuity that may be falsely interpreted as an occlusion. Nevertheless, both of these patients had poor outcomes (mRS scores of 5 and 6) and this was the reason why no other angiographic method was used for BAO confirmation. The lack of data regarding thrombus location and volume, etiology, and imaging characteristics, as well as the fact that physicians assessing the presence of BAO and recanalization were unblinded, represent other study limitations.

In conclusion, in this small acute BAO series, higher age, presence of diabetes mellitus, and presence of severe stroke were identified as significant independent negative predictors of good outcome, and a statistically insignificant trend for better clinical outcome was observed in patients treated with a combined IVT+IAT therapeutic approach. Limited randomized data on treating BAO are available, and there is a relative lack of clear guidelines for therapeutic choices. Currently, there is an ongoing prospective randomized BASICS trial that is devoted to the issues mentioned above, comprising evaluation of different treatment approaches. ${ }^{31}$

\section{ACKNOWLEDGEMENTS}

Study was partially supported by the grants of the Internal Grant Agency of Ministry of Health of the Czech Republic number NT/11046-6/2010, NT/11386-5/2010 and NT/13498$4 / 2012$, and by the grant project number CZ.1.05/2.1.00/01.0030.

\section{REFERENCES}

1. Israeli-korn SD, Schwammenthal Y, Yonash-Kimchi T, et al. Ischemic stroke due to acute basilar artery occlusion: proportion and outcomes. Isr Med Assoc J. 2010;12:671-5.

2. Mattle HP, Arnold M, Lindsberg PJ, Schonewille WJ, Schroth G. Acute basilar occlusion. Lancet Neurol. 2011;10:1002-14.

3. Gadoth A, Hallevi H. Basilar artery occlusion presenting as a tonicclonic seizure. Isr Med Assoc J. 2011;13:314-5.

4. Caplan LR. "Top of the basilar" syndrome. Neurology. 1980;30: 72-9.

5. Dornak T, Herzig R, Sanak D, Skoloudik D. Management of acute basilar artery occlusion: Should any treatment strategy prevail? Biomed Pap Med Fac Univ Palacky Olomouc Czech Repub. In press 2013 Sep 27. [Epub ahead of print]

6. Taneja SR, Hanna I, Holdgate A, Wenderoth J, Cordato DJ. Basilar artery occlusion in a 14-year old female successfully treated with acute intravascular intervention: case report and review of the literature. J Paediatr Child Health. 2011;47:408-14.

7. Chen MH, Wu SL, Lo MC, Chen WL, Wang WF. Basilar artery occlusion in a teenaged boy treated with intra-arterial thrombolysis. Acta Neurol Taiwan. 2010;19:281-6.

8. Goeggel Simonetti B, Ritter B, Gautschi M, et al. Basilar artery stroke in childhood. Dev Med Child Neurol. 2013;55:65-70.

9. Jung S, Mono ML, Fischer U, et al. Three-month and long-term outcomes and their predictors in acute basilar artery occlusion treated with intra-arterial thrombolysis. Stroke. 2011;42: 1946-51. 
10. Sairanen T, Strbian D, Soinne L, et al. Intravenous thrombolysis of basilar artery occlusion: predictors of recanalization and outcome. Stroke. 2011;42:2175-9.

11. Schonewille WJ, Wijman CA, Michel P, et al. Treatment and outcomes of acute basilar artery occlusion in the Basilar Artery International Cooperation Study (BASICS): a prospective registry study. Lancet Neurol. 2009;8:724-30.

12. Lindsberg PJ, Mattle HP. Therapy of basilar artery occlusion: a systematic analysis comparing intra-arterial and intravenous thrombolysis. Stroke. 2006;37:922-8.

13. European Stroke Organisation (ESO) Executive Committee; ESO Writing Committee. Guidelines for management of ischaemic stroke and transient ichaemic attack. Cerebrovasc Dis. 2008;25: 457-507.

14. European Stroke Organisation (ESO) Executive Committee; ESO Writing Committee. Should the time window for intravenous thrombolysis be extended? [Cited 2013 Jul 15] Available from: http://www.eso-stroke.org/pdf/ESO_Guideline_Update_ Jan_2009.pdf

15. Nagel S, Schellinger PD, Hartmann M, et al. Therapy of acute basilar artery occlusion: intraarterial thrombolysis alone vs bridging therapy. Stroke. 2009;40:140-6.

16. The National Institute of Neurological Disorders and Stroke rt-PA Stroke Study Group. Tissue plasminogen activator for acute ischemic stroke. N Engl J Med. 1995;333:1581-7.

17. Higashida RT, Furlan AJ, Roberts $\mathrm{H}$, et al. Trial design and reporting standards for intra-arterial cerebral thrombolysis for acute ischemic stroke. Stroke. 2003;34:e109-37.

18. Arnold M, Fischer U, Compter A, et al. Acute basilar artery occlusion in the Basilar Artery International Cooperation Study: does gender matter? Stroke. 2010;41:2693-6.

19. Hill MD, Kent DM, Hinchey J, et al.; PROACT-2 Investigators. Sex-based differences in the effect of intra-arterial treatment of stroke: analysis of the PROACT-2 study. Stroke. 2006;37: 2322-5.

20. Schulte-Altedorneburg G, Hamann GF, Mull M, et al. Outcome of acute vertebrobasilar occlusions treated with intra-arterial fibrinolysis in 180 patients. AJNR Am J Neuroradiol. 2006;27:2042-7.
21. Levy EI, Firlik AD, Wisniewski S, et al. Factors affecting survival rates for acute vertebrobasilar artery occlusions treated with intra-arterial thrombolytic therapy: a meta-analytical approach. Neurosurgery. 1999;45:539-48.

22. Greving JP, Schonewille WJ, Wijman CA, Michel P, Kappelle LJ, Algra A; BASICS Study Group. Predicting outcome after acute basilar artery occlusion based on admission characteristics. Neurology. 2012;78:1058-63.

23. Strbian D, Sairanen T, Silvennoinen H, Salonen O, Kaste M, Lindsberg PJ. Thrombolysis of basilar artery occlusion: Impact of baseline ischemia and time. Ann Neurol. 2013;73:688-94.

24. Vergouwen MD, Algra A, Pfefferkorn T, et al. Basilar Artery International Cooperation Study (BASICS) Study Group. Time is brain(stem) in basilar artery occlusion. Stroke. 2012;43: 3003-6.

25. Yu YY, Niu L, Gao L, et al. Intraarterial thrombolysis and stent placement for acute basilar artery occlusion. J Vasc Interv Radiol. 2010;21:1359-63.

26. Kashiwagi J, Kiyosue H, Hori Y, et al. Endovascular recanalization of acute intracranial vertebrobasilar artery occlusion using local fibrinolysis and additional balloon angioplasty. Neuroradiology. 2010;52:361-70.

27. Pfefferkorn T, Holtmannspötter M, Schmidt C, et al. Drip, ship, and retrieve: cooperative recanalization therapy in acute basilar artery occlusion. Stroke. 2010;41:722-6.

28. Lindsberg PJ, Soinne L, Tatlisumak T, et al. Long-term outcome after intravenous thrombolysis of basilar artery occlusion. JAMA. 2004;292:1862-6.

29. Barlinn K, Becker U, Puetz V, et al. Combined treatment with intravenous abciximab and intraarterial tPA yields high recanalization rate in patients with acute basilar artery occlusion. J Neuroimaging. 2012;22:167-71.

30. Mazighi M, Meseguer E, Labreuche J, Amarenco P. Bridging therapy in acute ischemic stroke: a systematic review and metaanalysis. Stroke. 2012;43:1302-8.

31. van der Hoeven E. BASilar artery International Cooperation Study (BASICS) trial. [Cited 2013 Jul 15] Available from: http:// basicstrial.com/Main.html. 